\title{
Protótipo de sistema para coleta de dados inerciais com foco na doença de Parkinson
}

\author{
Wanghley Soares Martins ${ }^{1}$, Fábio Henrique Monteiro Oliveira ${ }^{1}$ \\ ${ }^{1}$ Instituto Federal de Brasília - Campus Brasília (IFB) \\ SGAN Quadra 610 Módulos D, E, F, G \\ Asa Norte, Brasília - DF, 70830-450 \\ wanghleys@gmail.com, fabio.oliveira@ifb.edu.br
}

\begin{abstract}
Currently, the diagnosis and monitoring of people affected by neurodegenerative diseases are made subjectively. Parkinson's disease (PD), for example, is a neurodegenerative disease that affects the motor part of the individual. The evaluation of $P D$, usually is done through scales based on questionnaires. Thus, this research is dedicated to the study and development of a computational system capable of performing the collection, transmission and visual presentation of data related to the human movement, in order to assist the diagnosis and monitoring of PD.
\end{abstract}

Resumo. Atualmente, o diagnóstico e o acompanhamento de pessoas acometidas por doenças neurodegenerativas são feitos de maneira subjetiva. A doença de Parkinson (DP), por exemplo, é uma doença neurodegenerativa que afeta a parte motora do indivíduo. A avaliação da DP, normalmente é feita por meio de escalas baseadas em questionários. Dessa forma, a presente pesquisa dedica-se ao estudo e desenvolvimento de um sistema computacional capaz de realizar a coleta, transmissão e apresentação visual de dados relacionados ao movimento humano, a fim de auxiliar no processo de diagnóstico e acompanhamento da $D P$.

\section{Introdução}

De acordo com a Organização Mundial da Saúde, as doenças neurodegenerativas serão, em uma década, as doenças que mais deterioram a qualidade de vida de indivíduos com mais de 65 anos [Baumann 2012]. Os principais sintomas estão relacionados ao movimento do indivíduo, de forma que as atividades de vida diária são prejudicadas, tais como a escrita ou o manuseio de talheres. Paralelo ao mencionado, o uso de tecnologias vestíveis, os chamados wearables, está em crescente uso e é apontado como uma tendência mundial. Esses dispositivos vestíveis estão cada vez mais integrados ao cotidiano dos consumidores, incluindo indivíduos saudáveis, idosos e pessoas com doenças crônicas [Lauren C. Benson 2018].

Atualmente, o diagnóstico e o prognóstico de doenças neurodegenerativas, como a DP, são realizados por meio da observação de um neurologista apoiado por instrumentos subjetivos (e.g., escala unificada para avaliação da doença de Parkinson - UPDRS). Entre os sintomas mais comuns da DP está o tremor de repouso, que se manifesta nas extremidades periféricas corpórea do paciente, como as mãos e se inicial, normalmente, de forma unilateral ocorrendo em uma frequência que varia entre 4 e 6 Hz [Jankovic 2008]. 
Nesse sentido, este trabalho tem por objetivo apresentar um protótipo de sistema capaz de coletar e exibir dados de movimento da mão humana. Esse sistema, composto por hardware e software, almeja ser de baixo custo e capaz de coletar os movimentos da mão do indivíduo, e transmiti-los a um terminal de consulta do profissional da saúde. De forma a fornecer gráficos que traduzam os movimentos realizados pelo paciente.

\section{Materiais e Métodos}

O desenvolvimento deste trabalho foi dividido em três partes: (i) validação da ideia a nível de hardware; (ii) desenvolvimento de scripts para recebimento e plotagem dos dados de movimento; (iii) aprimoramento do hardware para compor um protótipo.

Na primeira parte, alguns sensores foram estudados e testados a partir do uso de plataformas embarcadas. Nesse processo, a partir de estudos dos sintomas comuns da DP, os sensores mais ideais para uso foram selecionados e relacionados ao movimento do indivíduo. Dessa forma, o primeiro protótipo de um dispositivo vestível capaz de realizar a coleta de dados inerciais foi desenvolvido.

Na primeira versão do sistema de coleta foram utilizados três sensores principais, (1) aceleração e (2) giroscópio, de forma a analisar o movimento realizado pelo paciente por meio do uso de conjuntos matemáticos denominados de quarteniões. Os quarteniões são utilizados para a representação gráfica de vetores e movimentos em um espaço cartesiano de três eixos (X, Y, Z). Os sensores utilizados foram controlados por um processador digital de movimento conhecido como MPU6050.

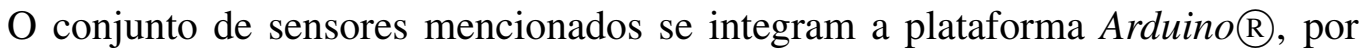
meio do Protocolo de Comunicação Entre Circuitos Integrados, do inglês Inter-Integrated Circuit $\left(I^{2} C\right)$. Dessa forma, os dados do sensor são digitalizados e enviados em tempo real, via Bluetooth. Os dados são então armazenados para a condução de análises.

Na segunda etapa foram desenvolvidos uma série de scripts - algoritmos computacionais - para recebimento, armazenamento e plotagem dos dados de movimento ${ }^{1}$. A Figura 2 ilustra a organização dos scripts.

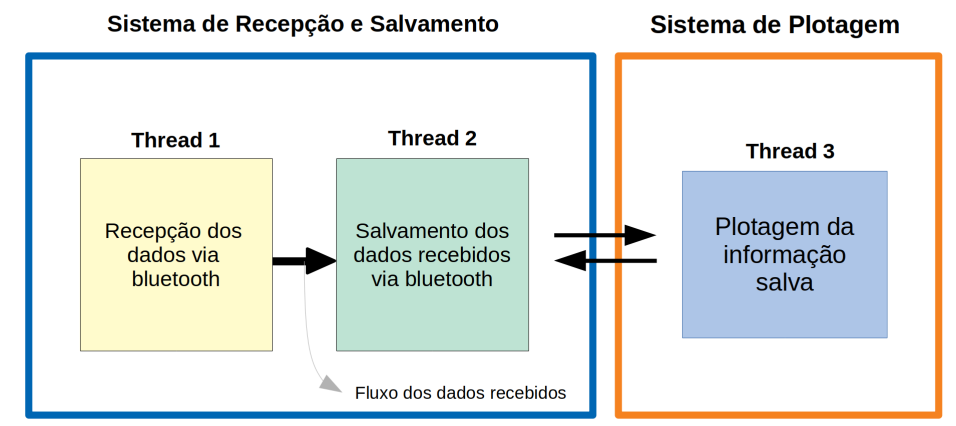

Figura 1. Organização do software para recepção, armazenamento e plotagem dos dados.

\footnotetext{
${ }^{1}$ repositório dos scripts criados disponíveis em: https:/github.com/Wanghley/PIBIC-Strategies-DataVisualization-Medicine
} 
O sistema de recepção, armazenamento e plotagem dos dados está organizado em três diferentes threads $^{2}$ (Figura 1). A primeira thread é responsável por estabelecer a conexão com o hardware, receber e gerenciar o fluxo de dados. A segunda thread é responsável por salvar os dados recebidos de forma consistente e organizada em arquivos no formato CSV. Por fim, a terceira thread é responsável por fazer a plotagem, em tempo real, dos dados aquisicionados.

Finalmente, na terceira etapa, o foco foi o aprimoramento do hardware, com o objetivo de minimizar interferência, mal contato etc. Para isso, uma placa de circuito impresso foi projetada e construída. A Figura 2 ilustra o circuito desenvolvido em software computacional de design eletrônico (a), junto ao hardware fabricado e montado com base no modelo desenvolvido (b).

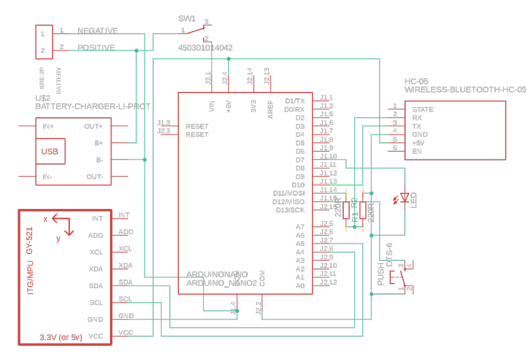

(A)

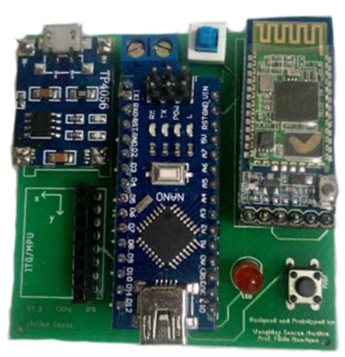

(B)

Figura 2. (a) Esquemático eletrônico e (b) hardware fabricado e montado.

Na Figura 3 é ilustrado os testes preliminares conduzidos. Os movimentos executados foram os seguintes: (1) pose do membro superior contra a gravidade formando aproximadamente noventa graus com o tronco; (2) desvio radial mantendo a pose descrita em (1); (3) desvio ulnar mantendo a pose descrita em (1). Entre cada atividade houve um repouso de três segundos [Keijsers 2003].

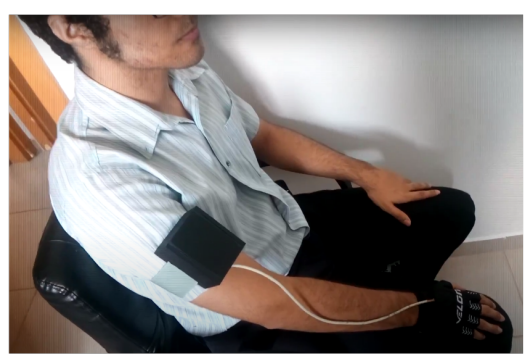

(a)

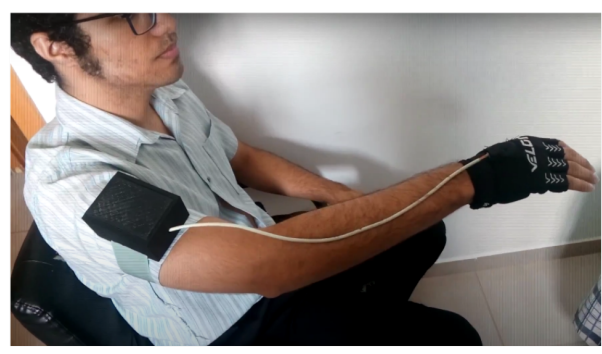

(b)

Figura 3. Teste preliminar do protótipo desenvolvido. Em (a) repouso e em (b) pose contra gravidade.

\section{Resultados e discussão}

Entre os principais resultados obtidos, está a integração no sistema de coleta, transmissão e análise de dados empregando os três pilares - (1) coleta, (2) transmissão e (3) plotagem

\footnotetext{
${ }^{2}$ threads são fluxos independentes de execução pertencentes a um mesmo processo, que requerem menos recursos de controle por parte do sistema operacional.
} 
dos dados - em uma única plataforma com resultados em tempo real da coleta realizada. Ademais, o sistema desenvolvido, por utilizar-se do protocolo $I^{2} C$, permite facilmente a adição de novos sensores.

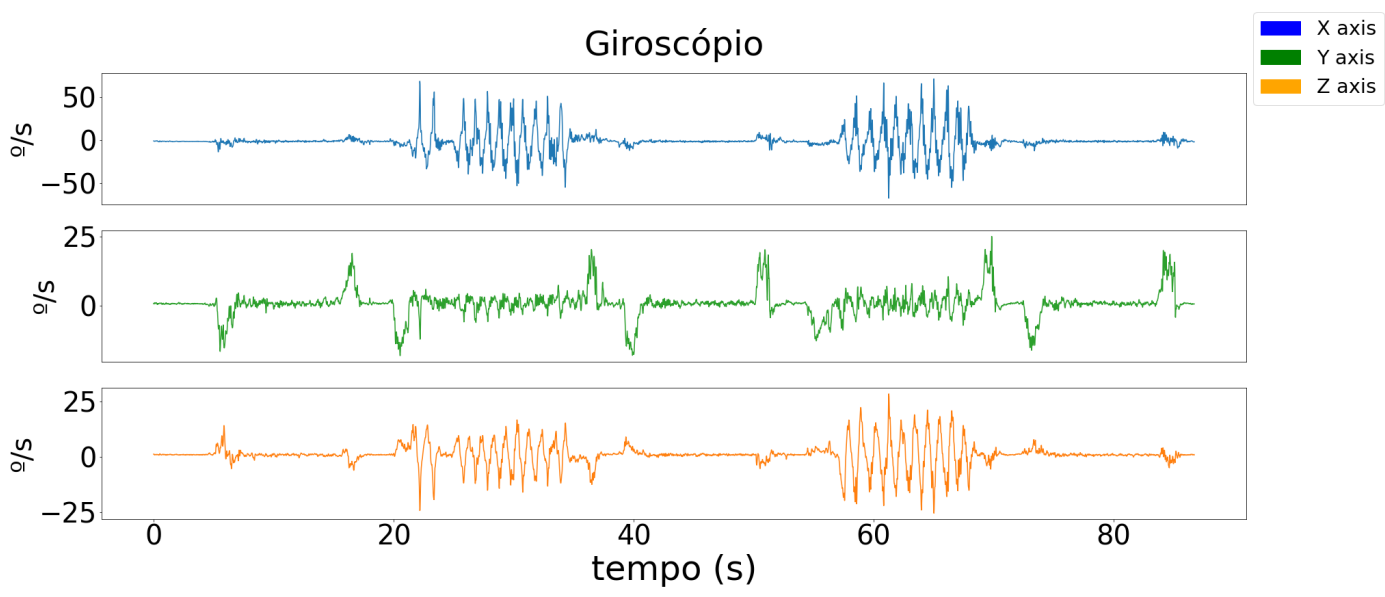

Figura 4. Exemplo de sinais adquiridos com o protótipo.

Na Figura 4 é observado os dados divididos de acordo com os eixos (X,Y e Z) da coleta realizada levando em conta os movimentos realizados. Observando o gráfico de aceleração do eixo X (esquerda em azul) da Figura 4 é possível visualizar a presença dos cinco movimentos realizados nos testes preliminares. Dessa forma, o sistema integra a coleta, transmissão e visualização da informação em um sistema único.

Todo o aparato montado, em funcionamento, possui custo médio de $\mathrm{R} \$ 230,00$, o que sugere que é possível a construção de um sistema capaz de realizar a coleta de dados inerciais em indivíduos com Parkinson por um valor acessível atingindo toda a população, inclusive a população periférica.

Por fim, o trabalho será expandido de forma a abordar os seguintes itens: (1) aprimoramento da integração entre hardware e software; (2) testes com indivíduos com e sem DP de forma a validar o equipamento.

\section{Referências}

Baumann, C. R. (2012). Epidemiology, diagnosis and differential diagnosis in parkinson's disease tremor. Parkinsonism and Related Disorders.

Jankovic, J. (2008). Parkinson's disease: clinical features and diagnosis. Journal of Neurology, Neurosurgery \& Psychiatry, 79:368-376.

Keijsers, N. L. W.; Horstink, M. W. I. M. . G. S. C. A. M. (2003). Online monitoring of dyskinesia in patients with parkinson's disease. Engineering in Medicine and Biology Magazine, pages 96-103.

Lauren C. Benson, Christian A. Clermont, E. R. (2018). The use of wearable devices for walking and running gait analysis outside of the lab: A systematic review. Elsevier. 